\title{
An Unusual Case of Encapsulating Peritoneal Sclerosis
}

\author{
S. Aloui ${ }^{a} \quad$ L. Jaafoura ${ }^{a} \quad$ R. Salem ${ }^{b} \quad$ M. Elmay ${ }^{a} \quad$ H. Skhiri

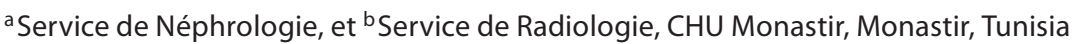

Encapsulating peritoneal sclerosis (EPS) is a rare complication of peritoneal dialysis and has an incidence rate of $1.8 / 1,000$ patient-years [1]. The usual clinical presentation is with partial or complete small bowel obstruction, ascites, abdominal mass, or impaired peritoneal ultrafiltration [2]. We report the case of a patient with EPS according to the original clinical and radiological presentation.

A 41-year-old man was admitted on December 29, 2009, for diarrhea and highgrade fever $\left(40^{\circ} \mathrm{C}\right)$. He began peritoneal dialysis in May 2001 for end-stage renal disease. He experienced three episodes of peritonitis which improved after treatment. The patient was transferred to hemodialysis therapy because of poor urea clearance and ultrafiltration on November 23, 2009. Examination revealed a distended abdomen, hypoactive bowel sounds, and diffuse abdominal tenderness. Investigations revealed no abnormality except CRP $300 \mathrm{mg} / \mathrm{l}$ and elevated serum creatinine. Abdominal radiography was performed (fig. 1) and showed extensive peritoneal curvilinear 'eggshell'-like calcification associated with vascular calcifications. The diagnosis of EPS was hardly suspected. Abdominal computed tomography showed dilated bowel loops with thickened walls, diffuse parietal and visceral peritoneal calcification, peritoneal thickening and enhancement, and encasement of the dilated bowel loops by the cal-

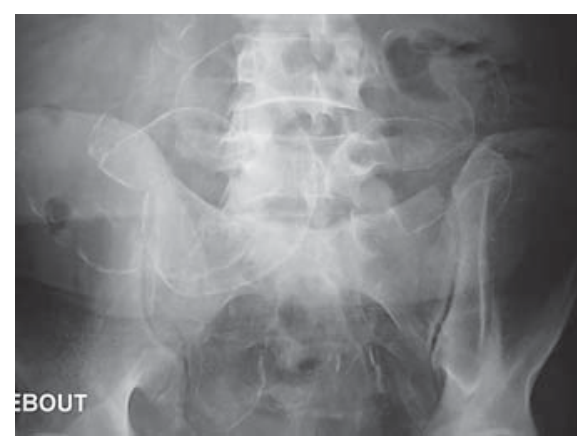

Fig. 1. Extensive peritoneal curvilinear 'eggshell'-like calcification associated with vascular calcifications.

cified peritoneum. Diagnosis of EPS was made. Steroids $(0.5 \mathrm{mg} / \mathrm{kg} /$ day $)$ and Tamoxifen ${ }^{\circledR} 20 \mathrm{mg}$ twice per day were started. The patient is now on a normal diet and CRP has returned to normal.

A definitive diagnosis of EPS is done at laparotomy or laparoscopy but may be strongly supported by a combination of clinical and radiologic features [1]. Computed tomography has largely been used to delineate more advanced disease. Abdominal X-ray is not useful but in our case, peritoneal calcification is prominent.

Risk factors are duration of peritoneal dialysis, peritonitis, poor biocompatibility of dialysate, acetate or lactate buffer, acidity, glucose, high osmolality, plasticizer, high transporter membrane [3].

Various treatment strategies are undertaken by various centers [4]. Steroids and immunosuppressive therapy remain the mainstay of treatment but we do not have enough information on when and for how long treatment would have to be used. Ultimately, parenteral nutritional support may be required along with surgical therapy to relieve intestinal obstruction [2]. EPS is rare and probably misdiagnosed although standard radiography can be very helpful.

\section{References}

1 Johnson DW, Cho Y, Livingston BE, et al: Encapsulating peritoneal sclerosis: incidence, predictors, and outcomes. Kidney Int 2010; 77:904-912.

2 Hidetomo N: Encapsulating peritoneal sclerosis a clinician's approach to diagnosis and medical treatment. Perit Dial Int 2005; 25(suppl 4):S30-S38.

3 Chin AI, Yeun JY: Encapsulating peritoneal sclerosis: an unpredictable and devastating complication of peritoneal dialysis. Am J Kidney Dis 2006;47:697-712.

-4 Balasubramaniam G, Edwina A, Brown EA, et al: The Pan-Thames EPS Study: treatment and outcomes of encapsulating peritoneal sclerosis. Nephrol Dial Transplant 2009;24: 3209-3215.

\section{KARGER}

Fax +41 613061234 E-Mail karger@karger.ch www.karger.com
S. Aloui

Service de Néphrologie

CHU Monastir

Monastir (Tunisia)

E-Mail alouisabra@yahoo.fr 